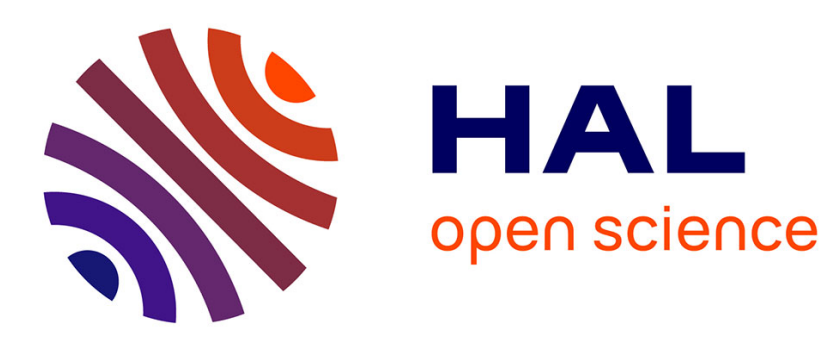

\title{
Optimization of the energy efficiency of a hybrid broadcast/unicast network
}

Nicolas Cornillet, Matthieu Crussière, Jean-François Hélard

\section{To cite this version:}

Nicolas Cornillet, Matthieu Crussière, Jean-François Hélard. Optimization of the energy efficiency of a hybrid broadcast/unicast network. Wireless Communications and Networking Conference Workshops (WCNCW), 2013 IEEE, Apr 2013, China. pp.39 - 44, 10.1109/WCNCW.2013.6533312 . hal00868915

\section{HAL Id: hal-00868915 https://hal.science/hal-00868915}

Submitted on 2 Oct 2013

HAL is a multi-disciplinary open access archive for the deposit and dissemination of scientific research documents, whether they are published or not. The documents may come from teaching and research institutions in France or abroad, or from public or private research centers.
L'archive ouverte pluridisciplinaire HAL, est destinée au dépôt et à la diffusion de documents scientifiques de niveau recherche, publiés ou non, émanant des établissements d'enseignement et de recherche français ou étrangers, des laboratoires publics ou privés. 


\title{
Optimization of the Energy Efficiency of a Hybrid Broadcast/Unicast Network
}

\author{
Nicolas Cornillet, Matthieu Crussière and Jean-François Hélard \\ Université Européenne de Bretagne, France \\ INSA, IETR, UMR 6164, F-35708 RENNES \\ Email: nicolas.cornillet@insa-rennes.fr
}

\begin{abstract}
In the last few years, mobile broadband communications operators have been confronted to an exponential growth of the traffic over their networks. Recently, the use of a broadcast component as a solution to face this growth has received a particular attention. In [1], a model for a hybrid network is introduced. This network consists in the inter-operation of a LTE unicast component and a DVB-T2 broadcast component. The introduced model allows the evaluation of the potential energy gain brought by such a network. In this paper, using an enhanced version of the model previously defined, we propose a new operating mode for the hybrid network that can improve the energy efficiency of the system.
\end{abstract}

\section{INTRODUCTION}

Since the end of the 20th century, telecommunication systems have engaged their digital revolution taking advantage of the high potential of digital signal processing and modulation technologies predicted by C.E. Shannon fifty years ahead. In the last nineties for instance, the first digital terrestrial television (DTV) broadcasts have been launched. Many countries are either currently in the last steps of their digital switch over or have already finished it. In the same time, broadband access technologies have also deeply mutated through the world wide intensive deployment of mobile cellular systems. By now, no fewer than four generations of cellular systems have already accompanied and brought fantastic changes in the habits and usages of telecommunication devices. As a result, mobile broadband operators are today confronted to a massive increase in data traffic, voice applications being paradoxically reduced to a small proportion of the cell phone usage. As this increase is not expected to stop in the next decade, digital dividend coming from DTV transition gives new perspectives to face the exploding data traffic demand.

One simple idea is off course to allocate the spectrum released in the process of analog TV switch off to the mobile broadband operators. This has partially been realized in many countries such as in the US where auction was carried out for the chunk of spectrum around 700MHz. However, since music, video and TV services are progressively entering portable or mobile devices such as smart phones or pads, it would certainly be worthful to take benefit from a broadcast access ensured by the already deployed DTV broadcast infrastructure.
In [1], we have led first investigations about the use of the freed broadcast spectrum in a mobile broadband perspective using broadcast towers and we have evaluated the potential gain of this solution from an energy point of view. By using a simple model of a hybrid network including a unicast component provided by the base stations of a cellular network and a broadcast component handled by the high-power DTV towers, we have evaluated the statistical distribution of the spectral efficiencies attainable by the users in the network. This allowed us to demonstrate the interest of the proposed solution since an energy gain was obtained for as few as one user every two unicast cells.

The present paper is an extension of this previous work. The first novelty of this paper is the use of a non-uniform distribution of the users in the network. This gives a more realistic representation of the actual distribution of the users and prevents from side effects encountered in the uniform model that was leading to user concentration at the edge of the broadcast coverage area. Moreover, such a refined model makes it possible to easily introduce a new mode of operation for the hybrid network where some users are covered by the broadcast component while the others are covered by the unicast component.

An additional novelty of this paper is the consideration of the difference between the two components in terms of Quality of Service (QoS). While the unicast component can rely on retransmissions to achieve a good quality of service, the broadcast component has to achieve the QoS through a single downlink transmission. The retransmission have an impact on the overall throughput of the system. We take this impact into account by defining the so-called effective spectral efficiency.

The rest of the paper is organized as follows. In section II the non-uniform user distributions and their influence on the system are presented. In section III, we discuss the influence of the retransmission mechanism used in the unicast component on the effective spectral efficiency. In section IV, an optimized operating mode for the hybrid network is introduced. Finally, we present simulation results before concluding. 


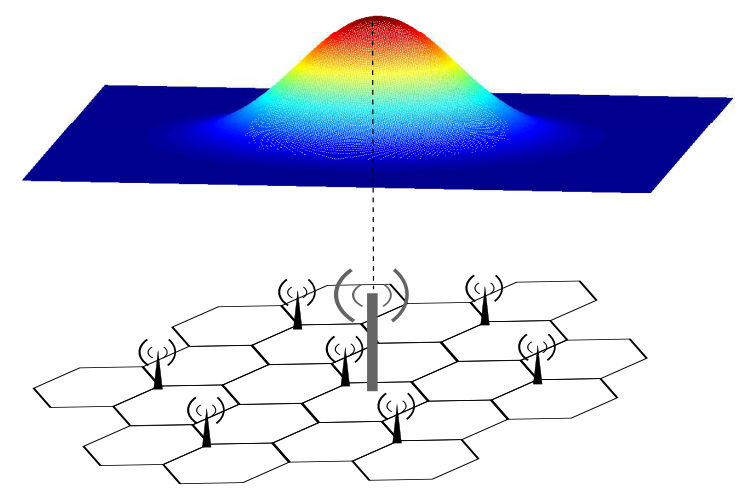

Fig. 1. Hybrid network model with Gaussian distribution of the users

\section{USERS DISTRIBUTIONS}

\section{A. Hybrid Network topology}

The hybrid network is the combination of two components. The first component is a classical cellular network. It consists of a great number of relatively small hexagonal cells. These cells are covered by low power base stations using a broadband signal such as LTE. The second component is a broadcast base station. Its coverage area is circular and overlaps the cellular network. The base station uses a high-power broadcast signal like recently normalized DVB-T2.

In this study, we consider a very simple propagation model. The channel under consideration is an Additive White Gaussian Noise (AWGN) channel and the attenuation is limited to the effect of the path loss.

The users location follows a 2 dimensional gaussian distribution as depicted Fig. 1. The distribution of the users will be used to evaluate the Probability Density Functions (PDF) of the distances between the users and the base stations and then the statistical properties of the spectral efficiencies that can be used.

\section{B. Centered gaussian distribution}

This type of distribution represents the case where the broadcast base station perfectly coincides with the center of the users distribution. Each user position is represented by two independent and identically distributed centered gaussian random variables $x$ and $y$. The PDF of the position of $x-y$ coordinates writes

$$
\operatorname{PDF}(x, y)=\frac{1}{\sigma^{2} 2 \pi} e^{-\frac{x^{2}+y^{2}}{2 \sigma^{2}}}
$$

In polar coordinates, this PDF becomes

$$
P D F(r, \theta)=\frac{1}{\sigma^{2} 2 \pi} e^{-\frac{r^{2}}{2 \sigma^{2}}}
$$

where $r=\sqrt{x^{2}+y^{2}}$ is the distance between a given user and the base station, and $\theta=\arg (x+i y)$. We can notice that this PDF only depends on the value of $r$.
The probability density function of $r$ is then calculated by

$$
\begin{aligned}
P D F(r) & =r \int_{0}^{2 \pi} P D F(r, \theta) d \theta \\
& =\frac{r}{\sigma^{2}} e^{-\frac{r^{2}}{2 \sigma^{2}}}
\end{aligned}
$$

\section{Non-centered gaussian distribution}

This type of distribution represents the case where the broadcast base station and the center of the gaussian distribution are not co-located. Each user position is represented by two independent non-centered gaussian variables $x$ and $y$. The center of the 2 dimensional gaussian distribution is represented by $x_{0}$ and $y_{0}$. The PDF of the position of $x-y$ coordinates writes

$$
P D F(x, y)=\frac{1}{\sigma^{2} 2 \pi} e^{-\frac{\left(x-x_{0}\right)^{2}+\left(y-y_{0}\right)^{2}}{2 \sigma^{2}}}
$$

In polar coordinates, this PDF becomes

$$
P D F(r, \theta)=\frac{1}{\sigma^{2} 2 \pi} e^{-\frac{r_{0}^{2}+r^{2}-2 r_{0} r \cos \left(\theta-\theta_{0}\right)}{2 \sigma^{2}}}
$$

where $r_{0}=\sqrt{x_{0}^{2}+y_{0}^{2}}$ is the distance between the base station and the center of the 2 dimensional gaussian distribution and $\theta_{0}=\arg \left(x_{0}+i y_{0}\right)$.

Let us state $\theta^{\prime}=\theta-\theta_{0}$. This allows us to write the probability density function of $r$,

$$
\begin{aligned}
P D F(r) & =r \int_{0}^{2 \pi} P D F(r, \theta) d \theta \\
& =r \int_{-\pi}^{\pi} \frac{1}{\sigma^{2} 2 \pi} e^{-\frac{r_{0}^{2}+r^{2}-2 r_{0} r \cos \left(\theta^{\prime}\right)}{2 \sigma^{2}}} d \theta^{\prime} \\
& =\frac{r}{\sigma^{2}} e^{-\frac{r^{2}+r_{0}^{2}}{2 \sigma^{2}}} I_{0}\left(\frac{r_{0} r}{\sigma^{2}}\right)
\end{aligned}
$$

where $I_{0}$ is the modified Bessel function of the first kind with order 0 .

\section{Users distribution within the unicast cells}

In the case of a non-uniform distribution of the users and following the same approach as in the case of a non-centered distribution within the broadcast cell, the PDF of the distance between a user and the base station in a unicast cell is

$$
P D F(r)=\frac{r}{\delta} \int_{-\theta_{H}(r)-\theta_{0}}^{\theta_{H}(r)-\theta_{0}} \frac{1}{\sigma^{2} 2 \pi} e^{-\frac{r_{0}^{2}+r^{2}-2 r_{0} r \cos \left(\theta^{\prime}\right)}{2 \sigma^{2}}} d \theta^{\prime}
$$

where $\theta_{H}(r)$ is the maximal angle within the cell at the distance $r$, as depicted Fig. 2, and $\delta$ is a normalization factor that represents the proportion of the number of users within a cell with respect to the total number of users in the overall hybrid network. Unfortunately, the 


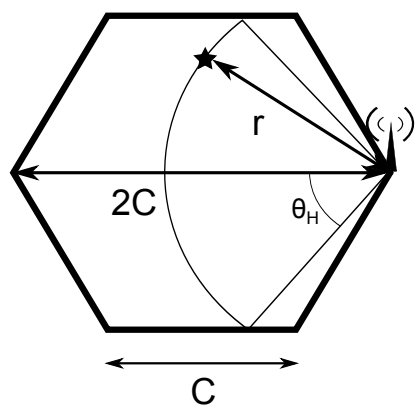

Fig. 2. Distance $r$ between the transmitter and one receiver in an hexagonal cell

fact that the integration domain is not $2 \pi$ makes the analytical evaluation of this expression quite difficult.

Let us restrict our study to the cases where $\sigma$ is of the same order of magnitude as the radius of the broadcast coverage area. Since this area is significantly larger than the unicast cell we can state that $\sigma$ is great relatively to $r$. Accordingly, we can state that the term $\frac{1}{\sigma^{2} 2 \pi} e^{-\frac{r_{0}^{2}+r^{2}-2 r_{0} r \cos \left(\theta^{\prime}\right)}{2 \sigma^{2}}}$ is almost constant on the area of an hexagonal cell. Let us denote this constant $\beta$, the expression of the PDF becomes

$$
P D F(r)=\frac{r}{\delta} \int_{-\theta_{H}(r)-\theta_{0}}^{\theta_{H}(r)-\theta_{0}} \beta d \theta^{\prime}=\frac{\beta}{\delta} 2 r \theta_{H}(r)
$$

By identification with the expression of the PDF of the distance found in [1] derived under the assumption of a uniform distribution of the users, we find that $\frac{\delta}{\beta}=A$, with $A$ being the area of the hexagonal cell. Finally, the PDF in the unicast case reads,

$$
\operatorname{PDF}(r)=\left\{\begin{array}{cl}
\frac{2 \pi \times r}{3 \times A} & r<C, \\
\frac{2 r \times \sin ^{-1}\left(\frac{\sqrt{3} C}{2 r}\right)}{A} & C<r<\sqrt{3} C, \\
\frac{2 r \times\left(\sin ^{-1}\left(\frac{\sqrt{3} C}{r}\right)-\frac{\pi}{3}\right)}{A} & \sqrt{3} C<r<2 C .
\end{array}\right.
$$

We verify the assumption made in the last paragraph through Monte Carlo simulations. The histogram given Fig. 3 represents the distribution of the distance between the users and the nearest base station, considering a Gaussian distribution of the users at the scale of the broadcast coverage area. We can notice that this histogram is similar to the red curve representing equation (9). We then conclude that the distribution of the users can reasonnably been considered as uniform at the scale of a unicast cell. Hence, from one unicast cell to another, the expression of the PDF given by (9) is valid and will remain the same. The only difference between cells rather comes from the number of users per cell which depends on the cell transmitter location. This assumption will be exploited in the sequel.

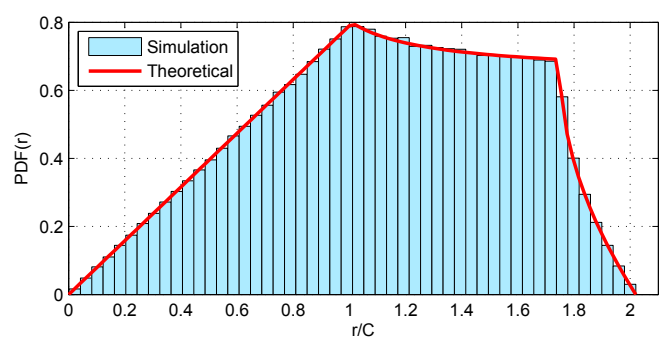

Fig. 3. Distribution of the distances within a unicast cell: comparison between the simulation results under Gaussian distribution and the theoretical results using uniform distribution

\section{SPECTRAL EFFICIENCIES DISTRIBUTION AND HARQ}

In order to provide a good quality of service, the two components of the system under consideration in our study rely on different mechanisms. The DVB-T2 standard, which is used for the broadcast component, does not have any retransmission mechanism. The required SNRs listed in [2] correspond to a transmission with a BER equal to $10^{-11}$ at the first transmission. The LTE standard, which is used for the unicast component relies on a retransmission mechanism named Hybrid Automatic Repeat reQuest (HARQ) [3] to achieve a quasi error free service. This mechanism has an impact on the effective spectral efficiency of the system which we intend to take into account hereafter.

\section{A. Distribution of the spectral efficiencies for the broadcast component}

For the broadcast component, since there is no retransmission mechanism, we evaluate the distribution of the spectral efficiencies with the same method as the one we used in [1]. Let $r_{i}$ be the furthest distance at which a user can receive properly the signal with a spectral efficiency of $\nu_{i}$,

$$
r_{i}=e^{\frac{1}{\alpha} \log \left(\frac{S N R_{\min }\left(\nu_{i}\right) r_{\max }^{\alpha}}{S N R_{\min }\left(\nu_{1}\right)}\right)}
$$

where $\nu_{1}$ is the smallest spectral efficiency available and $S N R_{\min }\left(\nu_{i}\right)$ is the minimal SNR required to achieve a proper reception of the signal with the spectral efficiency $\nu_{i}$. The probability for a particular spectral efficiency $\nu_{i}$ to be the best suited one for a particular user is then,

$$
P\left(\nu_{i}\right)=\int_{r_{i}}^{r_{i+1}} P D F(r)
$$

Applying this formula to all the available spectral efficiencies listed in the DVB-T2 specifications [2, chapter 14.2, table 45], we obtain the distribution depicted Fig. 4. 


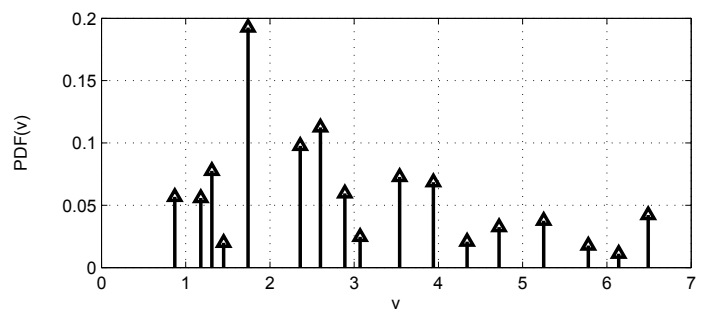

Fig. 4. PDF of $\nu$ for the DVB-T2 broadcast component under Gaussian distribution of the users in the coverage area.

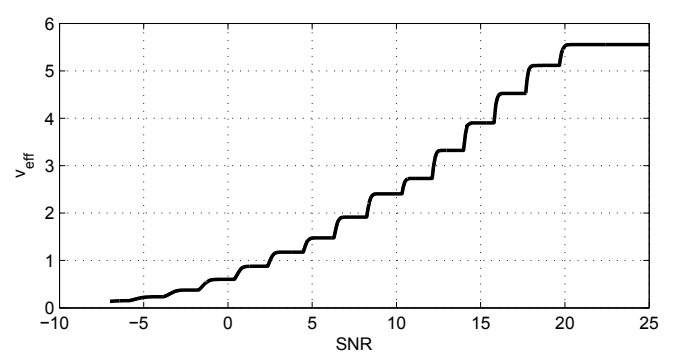

Fig. 5. $\nu_{\text {eff } f}$ as a function of $S N R$ for a LTE signal over AWGN channels

B. Distribution of the spectral efficiencies for the unicast component

We will represent the impact of the HARQ mechanism on the throughput in the form of the effective spectral efficiency $\nu_{\text {eff }}$.

$$
\nu_{e f f}=\frac{\nu}{T}
$$

where $T$ is the mean number of transmissions required to achieve a proper reception of a packet. More precisely, $\nu_{e f f}$ represents the spectral efficiency without any retransmission that would yield the same throughput as that obtained operating at spectral efficiency $\nu$ with an average number of transmissions $T$. The value of $T$ can simply be evaluated using block error rates (BLER) curves and can be expressed as,

$$
T=1+\sum_{i=2}^{\infty} \prod_{j=1}^{i-1} B L E R_{j}
$$

where $B L E R_{j}$ is the BLER after the $j^{\text {th }}$ transmission. Since our study is limited to AWGN channels and according to the steep descent of the LTE BLER curves due to turbo decoding, it is sufficient to only take into account the first two transmissions. Indeed, the use of HARQ on AWGN channels provide a quasi-error free communication with as few as two transmissions in the SNR areas of interest. Using the BLER values provided in [4], we can calculate the numerical values of $\nu_{e f f}=f(S N R)$ where $f$ is a function used in the following. This function is plotted in Fig. 5.

We consider that the cell is designed in order to achieve the minimal SNR, i.e. $S N R_{M I N}=-7 d B$

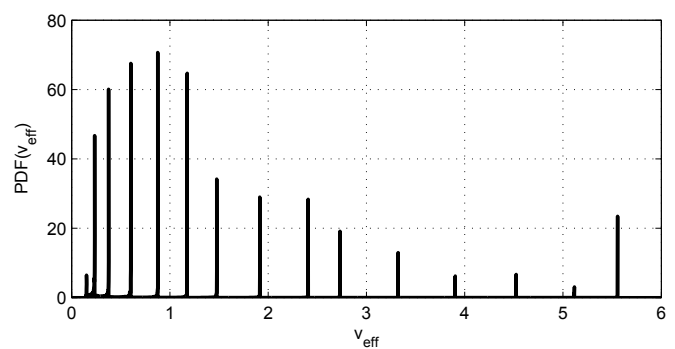

Fig. 6. Pobability Density Function of $\nu_{e f f}$ for the LTE unicast component

here, at its edge, i.e. for a distance $r_{M A X}$. This allows to express the SNR for any distance within the cell.

$$
S N R(r)=\left(\frac{r_{M A X}}{r}\right)^{\alpha} S N R_{\text {min }}
$$

By combining (14) with function $f$ previously defined, we find that

$$
\nu_{e f f}=f\left(\left(\frac{r_{M A X}}{r}\right)^{\alpha} S N R_{M I N}\right)
$$

From (9) we can hence evaluate the Cumulative Distribution Function (CDF) of $r$,

$$
C D F(R)=\int_{0}^{R} P D F(r) d r=g(R)
$$

Then, combining (15) and (16) allows us to express the $\mathrm{CDF}$ of $\nu$ as

$$
C D F\left(\nu_{e f f}\right)=g\left(r_{M A X}\left(\frac{f^{-1}\left(\nu_{e f f}\right)}{S N R_{M I N}}\right)^{\frac{1}{\alpha}}\right)
$$

Finally, the PDF of $\nu_{e f f}$, depicted in figure 6, is then obtained by

$$
P D F\left(\nu_{e f f}\right)=\frac{d}{d \nu_{e f f}} C D F\left(\nu_{e f f}\right)
$$

These expressions are used to evaluate the statistical properties of the effective spectral efficiencies available within a unicast cell. Note that the computation of the CDF and the PDF are achieved through numerical programming.

\section{MIXED OPERATING MODE}

In [1], we have only considered the case where the service was delivered to all the users through the same component of the system, unicast or broadcast. In this paper, we propose to investigate another case which consists in delivering the service to some users through the broadcast component and to other users through the unicast one. The underlying idea is to reduce the coverage of the broadcast base station to optimize its energy efficiency. The unicast network serves as an alternative link to deliver the service to the users who are no more covered by the broadcast network. 
Accordingly, we define three operating modes for the hybrid network : "Full Unicast", "Full Broadcast" and "Mixed".

In the "Full Unicast" mode, the service is delivered to all the users through the unicast component. As stated in [1], the energy consumption in this mode $E_{S}^{(U)}$ can be expressed as

$$
E_{S}^{(U)}=P S D_{U} \times Q_{S} \times \sum_{k=1}^{N} \frac{1}{\nu_{U, k}}
$$

with $U$ indicating unicast mode, $P S D_{U}$ being the Power Spectral Density (PSD) of the unicast signal, $Q_{S}$ being the weight of the service in bits, $N$ being the total number of users, $k$ being the user index $k \in[1 \cdots N]$, and $\nu_{U, k}$ being the maximal spectral efficiency allowing the user $k$ to properly receive the service.

In the "Full Broadcast" mode the service is delivered to all the users through the broadcast component. As stated in [1], the energy consumption in this mode can be expressed as

$$
E_{S}^{(B)}=P S D_{B} \times Q_{S} \times \max _{k \in[1 ; N]} \frac{1}{\nu_{B, k}}
$$

with $B$ denoting broadcast mode.

Finally, in the "Mixed" mode the set of users is divided into two subsets depending on the value of $\nu_{B, k}$. $K$ being the set of all the users, the two subsets are defined by

$$
K_{j}^{(B)}=\left[k \in K \mid \nu_{B, k} \geq \nu_{j}\right]
$$

and

$$
K_{j}^{(U)}=\left[k \in K \mid \nu_{B, k}<\nu_{j}\right]
$$

where $\nu_{j}$ is the $j^{t h}$ available spectral efficiency for the broadcast signal. In the following, we will designate the "Mixed" mode using a particular value of $\nu_{j}$ by the term "Mixed j" or $M_{j}$. The energy consumption in the mode "Mixed j" can be expressed as,

$$
\begin{aligned}
E_{S}^{\left(M_{j}\right)}= & Q_{S}\left(P S D_{B} \max _{k \in K_{j}^{(B)}} \frac{1}{\nu_{B, k}}\right) \\
& +Q_{S}\left(P S D_{U} \sum_{k \in K_{j}^{(U)}} \frac{1}{\nu_{U, k}}\right)
\end{aligned}
$$

\section{SIMULATION RESULTS}

To highlight the impact of the proposed hybrid network on performance from an energy consumption point of view, we define the energy gain $G_{U \rightarrow Y}$ obtained when using a particular mode $Y$ instead of the unicast mode, that is

$$
G_{U \rightarrow Y}=\frac{E_{S}^{(Y)}}{E_{S}^{(U)}}
$$

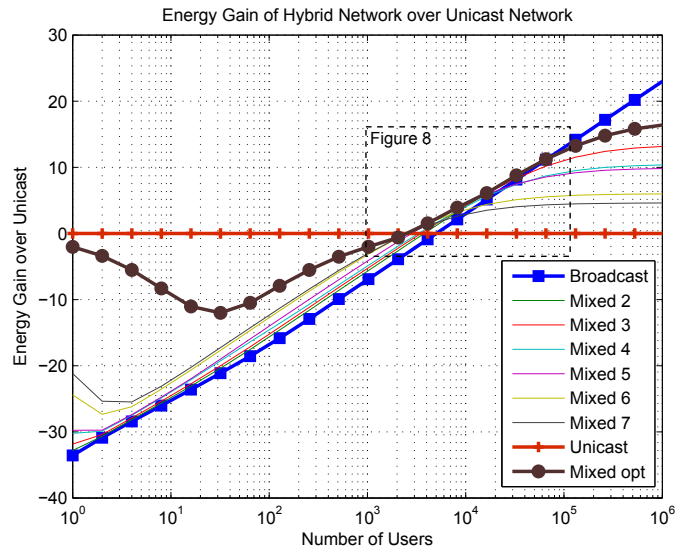

Fig. 7. Full simulation results (centered distribution)

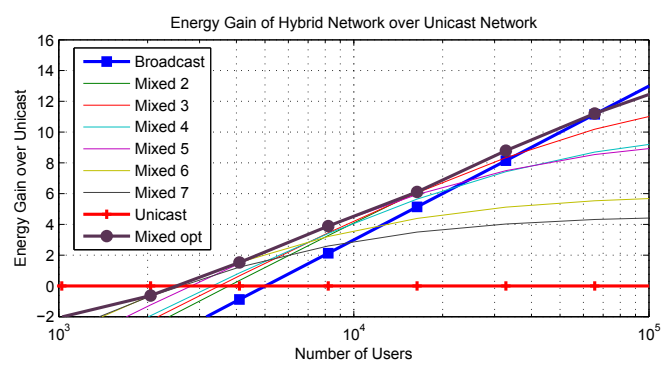

Fig. 8. Simulation results (centered distribution): zoom on the area of interest

\begin{tabular}{c|c|c} 
Parameter & Broadcast & Unicast \\
\hline$\alpha$ & 2.5 & 3 \\
\hline Frequency & $800 \mathrm{MHz}$ & $2.6 \mathrm{GHz}$ \\
\hline Cell radius & $\mathrm{R}=100 \mathrm{~km}$ & $\mathrm{C}=1 \mathrm{~km}$ \\
\hline User distribution & centered 2D gaussian \\
\hline$\sigma$ & \multicolumn{2}{|c}{$0.3 \mathrm{R}$} \\
TABLE I
\end{tabular}

Simulation results are depicted in Figs. 7 and 8 for the case of a centered Gaussian distribution of the users with respect to the location of the broadcast transmitter. Each curve represents the energy gain as a function of the number of users and using either the "Full Unicast", the "Full Broadcast" or various "Mixed" modes according to switching spectral efficiency values ranging from $\nu_{2}$ to $\nu_{7}$. Other parameters used for these simulations are listed in Table I.

From these results, we first note that the use of a broadcast component becomes more favorable in terms of energy consumption as soon as the number of users reaches a value around $5.0 \times 10^{3}$, and keeps increasing for an even denser network. This indicates that the amount of energy needed to spray all the broadcast coverage area remains lower than the sum of the energies required to serve each user, link by link, within each unicast cell. At the contrary, for a little number of users the unicast mode is prefered. Now considering the "Mixed" mode in the network, it appears that when the number of users reaches $2.4 \times 10^{3}$, a switch to the "Mixed" modes should be operated, the 


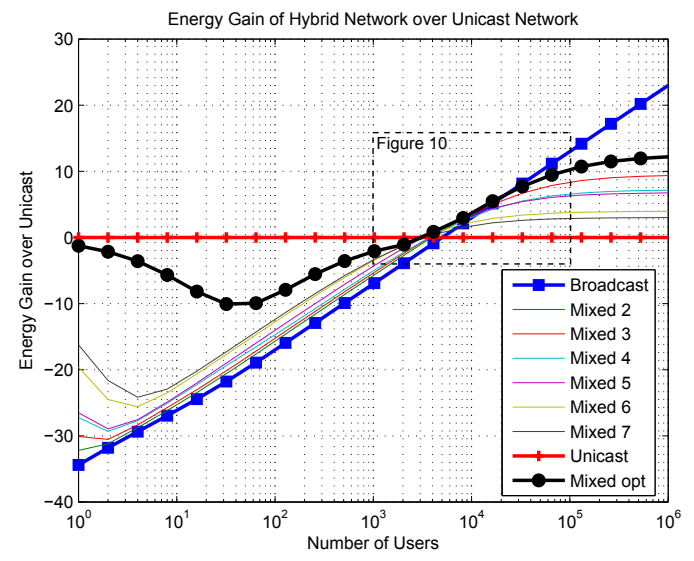

Fig. 9. Full simulation results(non centered distribution)

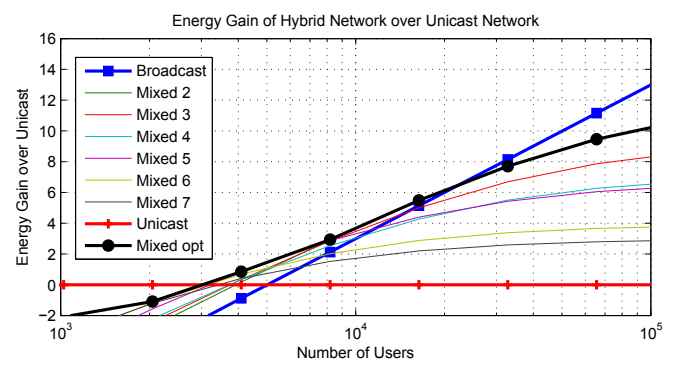

Fig. 10. Simulation results(non centered distribution): zoom on the area of interest

value of $j$ decreasing as the number of users increases. When the number of users go on increasing however, saturation floors are obtained for these "Mixed" modes. In particular, above $1.0 \times 10^{5}$ users, the hybrid network should operate in the pure broadcast mode. Finally, all these curves allow to choose the most efficient mode for any number of users, which leads to the optimal energy saving curve plotted with circle markers.

As a comparison to the results found in [1], we can notice that the threshold leading to energy gain over the unicast mode has been decreased from $5.0 \times 10^{3}$ users to $2.4 \times 10^{3}$ users. This clearly demonstrates the interest of the "Mixed" mode in this context. With the simulation parameters used here, the area of coverage of the broadcast component overlaps $1.2 \times 10^{4}$ unicast cells. This means that the use of the hybrid network provides an energy gain as soon as an average of 0.2 user per unicast cell are demanding access to a same service.

Fig. 9 and 10 depict the simulation results obtained for a non-centered 2D Gaussian distribution of the users. All others parameters are the same as listed in Table I. In this case, the center of the gaussian distribution is located 30 kilometers away from the broadcast base station. Interestingly, when comparing results of Figs. 8 and 10, we can notice that the switching point between unicast and broadcast modes is not influenced by the distribution of the users, its value being $5.0 \times 10^{3}$ for both cases. At the contrary, the interval of interest of the "Mixed" mode is greatly reduced compared to the centered case. Its lower bound moves from $2.4 \times 10^{3}$ to $3.2 \times 10^{3}$ and its upper bound moves from $1.0 \times 10^{5}$ to $2.2 \times 10^{4}$.

\section{CONCLUSION}

We have introduced an enhanced version of the system model previously proposed for unicast/broadcast hybrid networks including a more realistic distribution of the users and a better integration of the difference between LTE and DVB-T2 in terms of QoS. This new model allows to present a new mode of operation for the hybrid network, referred to as "mixed" mode. Through simulation runs, we conclude that the "mixed" mode is the most efficient strategy to reduce the energy consumption in a unicast/broadcast hybrid network. Nevertheless, its performance strongly depends on the distribution of the users, optimal results being achieved when the broadcast base station coincides with the high users density area. In the latter case and with the parameters used in our simulations, the use of the "Mixed" mode can provide energy efficiency improvement for as few as one user every five unicast cells.

\section{ACKNOWLEDGMENTS}

The authors would like to thank the French ANR project "M3" (Mobile Multi Media) and the "Images \& Réseaux" cluster for their support of this work.

\section{REFERENCES}

[1] N. Cornillet, M. Crussière, and J.-F. Hélard, "On the Hybrid Use of Unicast/Broadcast Networks Under Energy Criterion," Proceedings of IEEE 23rd International Symposium on Personnal, Indoor and Mobile Radio Communications (PIMRC 2012), Australia, Sydney, September 2012.

[2] ETSI TR 102 831, "Digital Video Broadcasting (DVB); Implementation guidelines for a second generation digital terrestrial television broadcasting system(DVB-T2)"

[3] Technical Specification Group Radio Access Network, "Evolved Universal Terrestrial Radio Access Network (E-UTRAN); Multiplexing and channel coding," 3rd Generation Partnership Project (3GPP), Tech. Rep.36.212 Version 11.0.0, September 2012

[4] C. Mehlfhrer, M. Wrulich, J.C. Ikuno, D. Bosanska, and M. Rupp, "Simulating the Long Term Evolution Physical Layer," Proceedings of 17th European Signal Processing Conference (EUSIPCO 2009), Scotland, Glasgow, August 2009. 